\title{
Assumed distance as a determinant of apparent size
}

\author{
FERN A. SINGER, ZITA E. TYER, and ROBERT PASNAK \\ George Mason University, Fairfax, Virginia 22030
}

\begin{abstract}
The hypothesis that size estimates could be systematically made when subjects were provided an assumed or perceived distance was tested. Forty subjects, each under one of four experimental conditions, were told the distance of a stimulus in a demonstratively cue-free situation. Their estimates of the size of the test objects constituted an increasing monotonic function of the size of the retinal image. Consistent overestimates of size suggest that the effect was primarily cognitive rather than perceptual.
\end{abstract}

Although many experiments report the effects of assumed or familiar size on the distance ascribed to a stimulus, there have been no tests of the converse. There is really no sound rationale as to why different retinal images $(\Theta s)$ used in conjunction with an assumed distance cannot be tested as an independent variable while reported size becomes the dependent variable. It may be, as Epstein $(1967$, p. 23) suggested, that the reason such a test has not been made is that objects have no "familiar distance," whereas they may have a "familiar size." There is no reason, however, that subjects cannot be given knowledge of an objects' distance and be asked to make size estimates on the basis of this knowledge in conjunction with $\Theta$. Whether subjects can do this in a systematic manner is an empirical question that can be answered by direct experimentation.

The present experiment was designed to be a methodological improvement over previous experiments. Oculomotor cue reduction was accomplished via a monocular pinhole eyepiece, which held accommodation constant, and stimuli were presented at relatively large distances. This eyepiece was mounted in the door of a huge room, so that there were no anchor cues from the size of the apparatus or experimental chamber. No reflecting surfaces were anywhere near the test object, there was no incandescent light source within the room, and subjects were not allowed to become dark adapted. Also, each subject viewed only one object, so relative size cues were eliminated.

An experimental design was used that provided a test of these cue reduction measures. In essence, stimuli having different sizes were presented at correspondingly different distances, so that they had the same $\Theta$. If size judgments were affected only by the distance that the subjects were told, they should have been identical for objects having the same $\Theta$. Also, if only the distance instruction affected size judgments, objects having

This research was supported in part by PHS Grant MH 17309 01. Reprint requests should be sent to Zita E. Tyer, Department of Psychology, George Mason University, Fairfax, Virginia 22030. different $\Theta$ s should have been judged equally different in size, whether the difference in $\Theta$ depended on physical distance or physical size. If, in constrast, uncontrolled distance cues were operating, size judgments would have varied not merely in accordance with $\Theta$, but also in accordance with physical distance. Thus a statistical assessment of cue reduction is available in this design.

\section{METHOD}

\section{Subjects}

Forty volunteers from undergraduate psychology and sociology courses at Catholic University, Washington, D.C., served as subjects in this experiment. Subjects were allowed to wear corrective lenses if necessary. A Bausch and Lomb Orthorater was used to test the visual acuity of each eye. The eye with the better visual acuity was used in the experiment. All subjects were required to have at least $20 / 25$ vision to participate in the experiment.

\section{Apparatus}

The experimental room with dimensions of $34.22 \times 14.63 \times$ $7.16 \mathrm{~m}$ was secured and made lighttight. The bottom of the door of the room was $1.45 \mathrm{~m}$ above floor level. A $15.24-\mathrm{cm}$-diam cavity was made in the door at a height of $1.37 \mathrm{~m}(2.82 \mathrm{~m}$ above the floor of the room), and a pinhole eyepiece designed to control accommodation was inserted.

Three aluminum disks with diameters of $2.54 \mathrm{~cm}, 5.08 \mathrm{~cm}$ and $10.16 \mathrm{~cm}$ painted with phosphorescent paint (Nite-lite by Rich Art) were suspended at eye level one at a time by black thread. Phosphorescence was activated with a $150-\mathrm{W}$ flood lamp held $30.32 \mathrm{~cm}$ away for $30 \mathrm{sec}$.

\section{Procedure}

Each subject was administered one of four randomly selected experimental conditions. Subjects viewed a $5.08-\mathrm{cm}$ disk at $1.52 \mathrm{~m}$, a $10.16-\mathrm{cm}$ disk at $3.04 \mathrm{~m}$, a $2.54-\mathrm{cm}$ disk at $3.04 \mathrm{~m}$, or a $5.08-\mathrm{cm}$ disk at $6.09 \mathrm{~m}$.

After the administration of the eye test, a subject was taken to the closed door housing the eyepiece. The location and orientation of the door made it difficult to estimate the dimensions of the experimental room. All subjects were instructed that the disk was $10 \mathrm{ft}$ away, thus providing a standard assumed distance for each subject. They were then asked to view a disk through the eyepiece and to estimate its size. Subjects' estimates were made in inches and were transformed to centimeters. 
Table 1

Objective Size, Means, and Standard Deviations of Estimates for the Four Experimental Conditions

\begin{tabular}{rcrlll}
\hline \multicolumn{3}{c}{ Stimulus } & & & \multicolumn{2}{c}{ Size Reports } \\
\cline { 1 - 3 } \cline { 6 - 6 } Size & Distance & ES & & Mean & SD \\
\hline 5.08 & 1.52 & 10.16 & & 22.61 & 16.43 \\
10.16 & 3.04 & 10.16 & & 22.61 & 15.54 \\
2.54 & 3.04 & 2.54 & & 14.48 & 10.09 \\
5.08 & 6.09 & 2.54 & & 10.67 & 10.94 \\
\hline
\end{tabular}

Note-Stimulus distances are given in meters; all other values are given in centimeters. $E S=$ equivalent size at $10 \mathrm{ft}$.

\section{RESULTS}

Means and standard deviations for each of the experimental conditions are found in Table 1 . Objects that had the same visual angle, $\Theta$, and were assumed to be at the same distance should have been reported as the same size. No test of significance was necessary in the case of the $5.08-\mathrm{cm}$ object at $1.52 \mathrm{~m}$ and the $10.16-\mathrm{cm}$ object at $3.04 \mathrm{~m}$, as the mean size reports were identical. There was no significant difference between the mean size reports for the $2.54-\mathrm{cm}$ object at $3.04 \mathrm{~m}$ and the $5.08-\mathrm{cm}$ object at $6.09 \mathrm{~m}[\mathrm{t}(18)=.95, \mathrm{p}>.05]$. Note that the direction of this nonsignificant difference is opposite to the true distance. This indicates that reduction of uncontrolled cues was complete enough that subjects could not detect the true differences in size or distance of the objects.

The hypothesis that size reports would be an increasing monotonic function of $\Theta$ when subjects were told the objects' distance was also supported by the data. This was true both when the physical size of the object was increased $[\mathrm{t}(18)=4.39, \mathrm{p}<.005]$, as in the case of the $2.54-\mathrm{cm}$ and $10.16-\mathrm{cm}$ stimuli at $3.04 \mathrm{~m}$, and when the distance of the object was increased $[\mathrm{t}(18)=2.93$, $\mathrm{p}<.005]$, as in the case of the $5.08-\mathrm{cm}$ stimuli at $1.52 \mathrm{~m}$ and $6.09 \mathrm{~m}$. The most parsimonious interpretation of these data is that as $\Theta$ increased, so did judged size according to the function size $/ 10=.0007 \Theta+.08$.

\section{DISCUSSION}

There are two possible interpretations of these data. One is that the distance instruction provided the basis by which subjects judged size. If this is the case, the effect would presumably be cognitive, because there is no known mechanism by which the distance instruction could be translated into a perception. While instructions that relate a stimulus to a familiar object might have a perceptual effect, because of the subjects' long experience with $\Theta$ s and distances of such objects, subjects have no corresponding experience with the $\Theta$ s and distances employed in this experiment. It appears that the subjects were able to relate $\Theta$ and the distance instruction relatively well, at least for the large $\Theta s$, since different subjects produced identical mean size reports. We note, however, that all sizes are greatly overestimated, which also suggests that the effect is cognitive. While overconstancy has sometimes been reported in full-cuenot reduced-cue-situations with objective size instructions (Carlson, 1960; Epstein, 1963), it has never approached this magnitude.

A second interpretation is that the specific distance tendency (SDT) caused the objects to appear 2-3 m distant (Gogel \& Tietz, 1973), overriding the distance instruction. If this is true, the size judgments would be perceptual, since the effect of the SDT is to produce perception both of distance and, consequently, of the size for any $\Theta$. Gogel (1976) argues that this perceptual effect would occur even when contradicted by cognitive information, with the resulting size judgments reflecting a compromise between the objects' familiar size and the cognitive information.

This explanation is unattractive for two reasons. First, the SDT shows up mainly when the head motion procedure is used to assess distance perception; the SDT has not had much effect on verbal reports of size or distance (Gogel, 1981; Gogel \& Tietz, 1973). More important, however, the direction of the error tendency in this experiment is opposite to that predicted for the SDT.

Thus, if operative, the SDT would result in all of the stimuli's being perceived as $2-3 \mathrm{~m}$ distant. Since this is closer than the actual distances of $10 \mathrm{ft}$ or of $20 \mathrm{ft}$, the SDT would have resulted in the stimuli's being perceived as nearer than they really were, and therefore, smaller than they really were. This is especially true for the stimulus presented at $20 \mathrm{ft}$. Since the obtained size reports are so strongly in the opposite direction, it seems inappropriate to conclude that the SDT influenced apparent distance.

The weight of the evidence, then, is that subjects can use cognitive information regarding the distance of unfamiliar objects to make systematic estimates of the objects' sizes. These estimates appear to be made primarily on a cognitive rather than a perceptual basis.

\section{REFERENCES}

Carlson, V. R. Overestimation in size-constancy judgments. American Journal of Psychology, 1960, 73, 199-213.

Epstein, W. Attitudes of judgment and the size-distance invariance hypothesis. Journal of Experimental Psychology, $1963,66,78-83$.

Epstein, W. Varieties of perceptual learning. New York: McGraw-Hill, 1967.

GOGEL, W. C. An indirect method of measuring perceived distance from familiar size. Perception \& Psychophysics, 1976, 20, 419-429.

Gogel, W. C. The role of suggested size in distance responses. Perception \& Psychophysics, 1981, 30, 149-155.

Gogel, W. C., \& Tietz, J. D. Absolute motion parallax and the specific distance tendency. Perception \& Psychophysics, 1973, 13, 284-292.

(Received for publication February 20, 1982.) 